\title{
\$) Nurbalacademic
}

\section{A vivência da maternidade em meio à pandemia}

The experience of motherhood during the pandemic

La experiencia de la maternidad en medio de la pandemia

Juliana Bernardo Silva Santos ${ }^{1}$ ORCID: 0000-0002-5079-8271

Emerson Santiago ${ }^{2}$

ORCID: 0000-0001-5173-7182

Elissandra Rodrigues Lopes ${ }^{1}$

ORCID: 0000-0002-4809-0186

Carla Merighi ${ }^{1}$

ORCID: 0000-0002-6322-0612

Aline Graziele Godoy Duarte ${ }^{1}$ ORCID: 0000-0002-2635-9770

Claudia Maria Silva Cyrino ${ }^{1}$

ORCID: 0000-0003-2442-2606

${ }^{1}$ Centro Universitário Sudoeste

Paulista. São Paulo, Brasil.

${ }^{2}$ Prefeitura de Itapetininga. São

Paulo, Brasil.

Como citar este artigo:

Santos JBS, Santiago E, Lopes ER, Merighi $C$, Duarte AGG, Cyrino CMS.

$A$ vivência da maternidade em meio à pandemia. Glob Acad Nurs.

2021;2(Spe.1):e95.

https://dx.doi.org/10.5935/2675-

5602.20200095

Autor correspondente:

Claudia Maria Silva Cyrino

E-mail: claucyrino@gmail.com

Editor Chefe: Caroliny dos Santos Guimarães da Fonseca

Editor Executivo: Kátia dos Santos Armada de Oliveira

Submissão: 19-01-2021

Aprovação: 27-01-2021

\section{Resumo}

Objetivou-se evidenciar os desafios da maternidade em meio à pandemia e descrever possíveis estratégias para vivenciar esse momento sem comprometer o campo afetivo-emocional do binômio. Para isso, realizouse uma pesquisa bibliográfica em bases de dados on-line com os descritores: pandemia, maternidade, trabalho doméstico, COVID-19. Com a inserção da pandemia pela COVID-19, encontram-se mães excepcionalmente atarefadas, na medida em que realizam o trabalho remoto em casa, encarregando-se das atividades domésticas e suprindo as necessidades dos filhos, que estão em tempo contínuo na residência. A redução de interação social expande o grau de estresse, ocorre uma disfunção no padrão do sono e aumenta os níveis de cortisol na corrente sanguínea. Em decorrência disso, emergiram efeitos negativos na saúde da população, tais como: ansiedade, medo, irritabilidade, alteração de apetite, dentre outros. A vista disso, o esgotamento físico e mental evidenciado torna-se reflexo no comportamento das crianças. Verificou-se a importância de um olhar atento para mães/mulheres que vivenciam a pandemia da COVID-19. Como estratégias, evidenciam-se: solicitar ajuda de familiares; divisão de tarefas e afazeres domésticos; apoio social; atividade física ou lazer; manter contato on-line com amigos/familiares; alimentação equilibrada; partilhar relatos de experiência; estabelecer uma pausa, mesmo que breve, para descanso.

Descritores: Infecções por Coronavírus; Maternidade; Pandemias; COVID-19.

\section{Abstract}

The aim was to highlight the challenges of motherhood during the pandemic and describe possible strategies to experience this moment without compromising the affective-emotional field of the binomial. For this, a bibliographic search was carried out in online databases with the descriptors: pandemic, maternity, domestic work, COVID-19. With the insertion of the pandemic by COVID-19, mothers are exceptionally busy, as they perform remote work at home, taking care of domestic activities and meeting the needs of their children, who are in continuous residence. The reduction in social interaction expands the degree of stress, there is a dysfunction in the sleep pattern and increases the levels of cortisol in the bloodstream. As a result, negative effects on the population's health emerged, such as: anxiety, fear, irritability, changes in appetite, among others. In view of this, the physical and mental exhaustion evidenced becomes a reflection in the children's behavior. It was verified the importance of a close look at mothers / women who experience the COVID-19 pandemic. As strategies, they are evidenced: asking for help from family members; division of tasks and household chores; social support; physical activity or leisure; maintaining online contact with friends / family; balanced diet; share experience reports; establish a break, however brief, for rest.

Descriptors: Coronavirus Infections; Maternity; Pandemic; COVID-19.

\section{Resumén}

El objetivo fue resaltar los desafíos de la maternidad en medio de la pandemia y describir posibles estrategias para vivir este momento sin comprometer el campo afectivo-emocional del binomio. Para ello, se realizó una búsqueda bibliográfica en bases de datos en línea con los descriptores: pandemia, maternidad, trabajo doméstico, COVID-19. Con la inserción de la pandemia por COVID-19, las madres se encuentran excepcionalmente ocupadas, ya que realizan trabajos a distancia en el hogar, ocupándose de las actividades domésticas y atendiendo las necesidades de sus hijos, quienes se encuentran en residencia continua. La reducción de la interacción social amplía el grado de estrés, hay una disfunción en el patrón de sueño y aumenta los niveles de cortisol en el torrente sanguíneo. Como resultado, surgieron efectos negativos en la salud de la población, tales como: ansiedad, miedo, irritabilidad, cambios en el apetito, entre otros. Ante esto, el agotamiento físico y mental evidenciado se convierte en un reflejo en el comportamiento de los niños. Se verificó la importancia de observar de cerca a las madres / mujeres que viven la pandemia de COVID-19. Como estrategias se evidencian las siguientes: pedir ayuda a los familiares; división de tareas y quehaceres domésticos; apoyo social; actividad física u ocio; mantener el contacto en línea con amigos / familiares; dieta equilibrada; compartir informes de experiencias; establezca una pausa, aunque sea breve, para descansar.

Descriptores: Infecciones por Coronavirus; Maternidad; Pandemia; COVID-19. 
A vivência da maternidade em meio à pandemia

\section{Introdução}

Maternidade não se refere a um acontecimento biológico, mas à uma vivência inscrita numa dinâmica sóciohistórica. Implica na prestação de cuidados, envolvimento afetivo em medidas variáveis. Mas, a vivência de ambas depende não só das características individuais de cada mulher, mas também do seu enquadramento sóciohistórico ${ }^{1}$.

Todavia, a forma como é vivenciada a maternidade compara-se a uma metamorfose, porquanto se altera em função das exigências e dos valores que dominam numa determinada sociedade e em num determinado momento ${ }^{1}$.

O início do ano de 2020 foi sinalizado por um surto de uma variação da COVID-19, no qual o primeiro caso foi confirmado em dezembro de 2019 na cidade de Wuhan, na China. Em decorrência disso, a Organização Mundial de Saúde (OMS) declarou situação de emergência em saúde pública de interesse internacional, ao final de janeiro de $2020^{2}$.

Não obstante, em 11 de março do mesmo ano, a OMS declara a COVID-19 como pandemia e estabelece medidas indispensáveis para a prevenção e enfrentamento deste novo cenário. Dentre essas medidas estão: higienizar as mãos com água e sabão, o hábito do uso de álcool em gel em momentos que é inviável a lavagem das mãos, evitar tocar olhos, nariz e boca, etiqueta respiratória. Ademais, a OMS recomenda-se o distanciamento social (mínimo de um metro), utilizar máscara, evitar aglomerações e medidas ambientais $^{2,3}$.

A doença COVID-19 teve uma rápida disseminação e, como decorrência, instituições governamentais de saúde mobilizaram planos de prevenção, urgência e emergência para conter o aumento exponencial de casos, a fim de evitar a sobrecarga nos serviços de atendimento à população ${ }^{4}$.

A transmissão acontece de um indivíduo infectado para outro, ou por contato próximo por meio de: toque, aperto de mãos contaminadas, gotículas de saliva, espirros, tosse, secreção de vias aéreas superiores, objetos ou superfícies contaminadas, como celulares, mesas, talheres, maçanetas, brinquedos, teclados de computador entre outros $^{5}$.

Os critérios utilizados para conter o vírus causaram um grande impacto na economia, relações sociais, convivência familiar e, por conseguinte, na saúde mental das pessoas. Insere-se, de fato, no contexto do desenvolvimento capitalista inicial, que retirou a mulher do espaço laboral, remetendo-a ao domicílio para realização de trabalho reprodutivo não remunerado 4 .

Pesquisadores $^{6}$ abordam em seu estudo a existência de outras pandemias que ocorreram entre os séculos XIV e XXI, dentre elas estão: influenza 1889-1890, gripe espanhola 1918-1920, gripe asiática 1957-1958, gripe de Hong Kong 1968-1969, gripe russa 1977-1978, gripe aviária 2003-2004, sendo a última a pandemia de 2009 por H1N1.

Refletir sobre os aspectos psicológicos da maternidade leva a reconhecer acerca da sobrecarga de trabalho acumulado pelas mulheres, especialmente as mães, com a intensificação do convívio familiar no contexto do
Santos JBS, Santiago E, Lopes ER, Merighi C, Duarte AGG, Cyrino CMS isolamento social, proposto como ação de contenção da infecção pela COVID-19, motiva uma reflexão sobre a espacialidade e a densidade das relações domésticas ${ }^{7}$.

Analisar a maternidade, inserida no contexto da pandemia, acarreta a realização de trabalho profissional em ambiente doméstico e a permanência contínua de crianças em seus lares, amplia-se a compreensão sobre as repercussões subjetivas da dupla jornada, também conhecida como segundo turno ${ }^{4}$.

Contudo, mesmo a mulher profissionalmente ativa, passa a afigurar-se como a principal e melhor cuidadora, como se pode notar empiricamente. Desse modo, uma evidente sobrecarga que gera sofrimentos emocionais, socialmente determinados, e que atingem tanto as próprias mães como os filhos e demais familiares ${ }^{4}$.

É substancial refletir sobre a maternidade em meio à pandemia da COVID-19, bem como a importância do cuidado profissional somado ao apoio psicológico e orientações sobre prevenção e cuidados. A suspensão das aulas e o fechamento das escolas somam-se a novas formas de estresse aos cuidadores, tornando-os, por vezes, incompatíveis com outras tarefas ${ }^{7}$.

Autores $^{8}$ ressaltam que, em meio ao caos e incerteza do amanhã, apoiar uma mulher-mãe em tempos da COVID-19, pode salvá-la de uma situação angustiante. Assim sendo, sustentar, amparar, firmar, auxiliar, ajudar, todas essas ações e outras tantas afins são capazes de confortar um indivíduo. Pode-se buscar, também, este amparo virtualmente, onde existem grupos em redes sociais, tais como Whatsapp ${ }^{\circledR}$ e Facebook $^{\circledR}$, entre outros aplicativos, com o intuito de acolhimento.

Destaca-se, também, a importância da rede de apoio formada por grupo de familiares, amigos, vizinhos, profissionais. Essas redes são capazes de promover uma ajuda emocional (expressões de conforto e cuidado), informacional (informações e orientações) ou instrumental (provisão de recursos, serviços e solução de problemas) ${ }^{9}$.

Em contrapartida, por vezes, a presença masculina permanente no lar não significa cooperação ou distribuição justa das tarefas entre toda a família, mas sim o aumento do trabalho invisível e não remunerado das mulheres. 0 trabalho doméstico se torna maior e mais exaustivo à medida em que há mais pessoas em continuidade na residência ${ }^{10}$.

De acordo com o IBGE, 2017, 83,7\% das crianças menores de 4 anos tinham uma mulher como primeira pessoa responsável por elas, enquanto apenas $16,3 \%$ tinham homens como principal responsável. Dessas crianças, $46 \%$ estavam sob a responsabilidade de uma mulher "dona de casa" e $37 \%$ delas por mulheres que exercem atividades laborais, portanto, nota-se uma condição de sobrecarga com maiores proporções entre as mulheres ${ }^{7}$.

Estudo $^{7}$ refere que a suspensão das aulas e o fechamento das escolas adicionaram novas formas de estresse aos cuidadores e, em muitos casos, tornou este cuidado inconciliável com outros. Em sua pesquisa, afirmou que, com a pandemia, a divisão sexual do trabalho, que já era desigual, teve a tendência de se agravar. Mesmo no caso de pesquisadoras e cientistas, trabalhadoras privilegiadas 
A vivência da maternidade em meio à pandemia

em termos de garantia da renda frente à maior parcela das mulheres-mães, manter-se produtiva acadêmicacientificamente, sem comprometer a qualidade do campo afetivo-emocional do binômio, tornou-se um desafio.

Diante do exposto acima e a relevância que o tema apresenta na atualidade é que se propõe a realização desse estudo a partir da seguinte questão norteadora: Qual o impacto da pandemia na maternidade? Assim, o objetivo do estudo foi evidenciar os desafios da maternidade em meio à pandemia e descrever possíveis estratégias para vivenciar esse momento sem comprometer o campo afetivoemocional do binômio.

\section{Metodologia}

Trata-se de um estudo descritivo de revisão bibliográfica.

As fontes utilizadas como recursos de pesquisa e coleta de dados foram as bases de dados on-line: Google Acadêmico, Biblioteca Virtual em Saúde, SciELO, dentre outros, para busca de artigos com as palavras-chaves: "Pandemia", "Maternidade", "Trabalho Doméstico", "COVID-19".

Foram selecionados artigos científicos completos no idioma português, assim como, portarias ministeriais sobre o tópico apresentado, sem determinação de tempo.

A coleta de dados nas bases citadas foi realizada entre os meses de fevereiro e julho de 2020.

Para análise dos textos selecionados, foram identificadas ideias centrais que nortearam a pesquisa, como demonstrar as dificuldades maternais enfrentadas, a importância da maternidade em meio à pandemia, bem como propor formas/estratégias de vivenciar esse período pandêmico sem prejuízos ao binômio.

\section{Resultados e Discussão}

Em um cenário que é necessário permanecer no lar por tempo indeterminado, é portado como privilégio aos que podem "se dar ao luxo" de não sair e, todavia, conjuntamente é exaustivo em termos emocionais quando não parece haver outra saída de evitar a propagação do vírus ${ }^{7}$.

As mulheres que estão na base dos serviços considerados essenciais são as que encontram maiores dificuldades por terem que acumular funções, somando-se às rotinas fora de casa, permitem que seus filhos dependam do convívio com parentes ou pessoas próximas à família, expondo-se aos riscos de infecção pela COVID-19. Demasiadas são as reportagens ou postagens em redes sociais, com centenas de milhares de comentários e compartilhamentos, abordando o tema da exaustão materna na pandemia ${ }^{7}$.

Nessa perspectiva, encontram-se mães excepcionalmente atarefadas, na medida em que estão realizando o trabalho remoto em casa, encarregando-se das atividades domésticas e suprindo as necessidades dos filhos, que estão em tempo contínuo na residência.

Autores $^{11}$ sugerem algumas orientações para amenizar e deixar sutil esta vivência. Eles afirmam que combinar diversas atribuições gera exaustão e ansiedade.
Santos JBS, Santiago E, Lopes ER, Merighi C, Duarte AGG, Cyrino CMS Ademais, a mãe/mulher não precisa dar conta de tudo, uma vez que, pode resultar em sobrecarga mental. Em contrapartida, o estudo mostra algumas estratégias que podem ser capazes de amenizar este impacto na maternidade, como por exemplo, solicitar e aceitar ajuda dos familiares, orientar a dividir os encargos e tarefas, desse modo, que todos possam ajudar e partilhar a criar novos hábitos.

Estudo $^{12}$ evidencia que os indivíduos desfrutem do confinamento para colocar as atividades domésticas em dia, fortalecer ou não os laços familiares, estudar, ler, ouvir música, assistir filmes ou realizar quaisquer atividades, a fim de melhorar a qualidade de vida em situação de isolamento. Contudo, para alguns pais, é esgotante permanecer com os filhos em casa em tempo total, uma vez que, eles estão acostumados ao ritmo frenético da vida urbana e uma rotina desapegada com os que mantém relação afetiva.

Estudo $^{13}$ afirma que a redução de interação social expande o grau de estresse, ocorre uma disfunção no padrão do sono e aumenta os níveis de cortisol na corrente sanguínea. Em decorrência desta pandemia, emergem efeitos negativos na saúde da população, tais como: ansiedade, medo, irritabilidade, alteração de apetite e aumento do consumo de álcool, tabaco e drogas.

Nesse sentido, o Center for Disease Control and Prevention (CDC), dos Estados Unidos, preconiza algumas orientações a fim de reduzir os efeitos colaterais da pandemia, dentre eles estão: assegurar contato on-line com amigos e família, praticar exercícios físicos, técnicas de relaxamento e manter uma alimentação equilibrada. Além disso, é necessário que as informações compartilhadas sobre a COVID-19 corroborem com fontes confiáveis. À vista disso, o esgotamento físico e mental evidenciado no adulto, tornase reflexo no comportamento das crianças ${ }^{13}$.

A mãe/mulher não precisa ser em tempo integral a única responsável pelas crianças, em alguns momentos, dentro da realidade de cada um, deve-se solicitar apoio às crianças mais velhas que tenham capacidade de observar e entreter as crianças mais novas, a fim de ambas desfrutarem de momentos de diversão e cuidados. É preciso desnaturalizar a ideia das mulheres como principais cuidadoras $^{7,11}$.

Desfrutar o lar como um ambiente seguro, de descanso e proteção deveria ser um direito básico garantido, mas na prática ainda é um privilégio de classe e de gênero ${ }^{10}$.

Cabe ainda ressaltar um estudo ${ }^{14}$ que a aborda a terapia cognitiva comportamental, por meio da psicoeducação, a qual torna-se uma estratégia efetiva para a população em geral, quando associada a técnicas de relaxamento e respiração para o controle da ansiedade, favorecendo a manutenção da saúde mental e física.

Somando-se a isso, o apoio social surge como uma ferramenta de proteção que ajuda os indivíduos a enfrentarem situações de esgotamento mental. Por isso, ressalta-se a importância de usar outras possibilidades não presencias com o intuito de fortalecer o vínculo do apoio social, por meio do encontro remoto com ligações telefônicas e chamadas de vídeo ${ }^{14}$. 
$A$ vivência da maternidade em meio à pandemia

No entanto, a população de classes média e alta desfruta de alguns passatempos, o que difere de pessoas de classe baixa que não tem esse mesmo acesso. Dado que, indivíduos que residem em lugares pequenos, poucos recursos e com maior número de pessoas, têm maior dificuldade em aderir estas estratégias, pois muitos não têm acesso aos aplicativos ou conexão à Internet e, com isso, devido à estrutura da moradia, por vezes, é inviável realizar atividades relaxantes, divertidas ou qualquer prática de atividade física ${ }^{13}$.

Para autores ${ }^{11}$, é preciso organizar intervalos para o seu próprio repouso e fazer o que lhe traz satisfação, como por exemplo, meditar, ler, ouvir música, conversar por meios eletrônicos ou praticar algum exercício físico. Mesmo que esses intervalos não sejam por longos períodos, é necessário e benéfico, e será notável a diferença para todos. Estudo ${ }^{14}$ corrobora com esse achado quando afirma que a prática regular de atividade física auxilia na regularização do sono e controle da ansiedade.

Fundamenta-se a capacidade das crianças auxiliarem nas atividades domésticas de acordo com a sua idade, a fim de estimular a autonomia, responsabilidade e senso de organização. Pode-se orientar a criança a guardar os brinquedos, arrumar o quarto, sendo assim, todos os membros contribuem com a manutenção do lar e redução da sobrecarga materna ${ }^{11}$.

Algumas estratégias recomendadas por autores ${ }^{15}$ à população estão ofertadas em formato de cartilhas, materiais informativos, canais para escuta psicológica, com o objetivo de atenuar sentimentos negativos. Os atendimentos on-line têm ocorrido para compreender o estado de saúde mental da população frente à pandemia da COVID-19. Ainda que, de forma longínqua, propõe-se oferecer cuidados psicológicos, os quais consistem em assistência e amparo em situações de crise, reduzindo, assim, o estresse, proporcionando conforto e ativando a rede de apoio social, uma vez que os sentimentos de ansiedade, tristeza, medo, solidão e raiva são esperados no contexto de pandemia.

Partilhar relatos de experiências em momentos de turbulência desenvolve um senso de universalidade e traz sensações de alívio. A presença das redes de apoio social antes, durante e após situações de grande impacto elevam a viabilidade de se manter a saúde mental após uma crise. Portanto, sugestões para a manutenção da saúde mental necessitam ser divulgadas vastamente. A divulgação pode ser feita em canais de rádio e televisão, mídias sociais ou carros de som ${ }^{13}$.

Autores $^{16}$ retratam em seu estudo que podem ocorrer culpas e incertezas no dever de desempenhar um papel social decorrente de um processo de socialização em que meninas e meninos interiorizam o mundo social como sendo a realidade objetiva. É nesse processo que se assimila um acontecimento objetivo como fadado de coerência, tornando subjetivamente o significado "ser mulher" e "ser mãe", interiorizando fortemente na mulher/mãe, a qual sente-se na obrigação de seguir um padrão ideal de mãe, de modo que quando não atingido da forma como é expectável pela sociedade em geral, gera-se a auto-culpa.
Santos JBS, Santiago E, Lopes ER, Merighi C, Duarte AGG, Cyrino CMS

$\mathrm{Na}$ contemporaneidade, o trabalho doméstico, formal ou não, é de modo específico atribuído às mulheres, representando uma desigualdade entre os gêneros masculino e feminino. Esse trabalho é sinalizado por dor, opressão e adoecimento. Sistematicamente, a própria mulher internaliza nas relações, a obrigação desses afazeres, e se isenta para cuidar de si mesma, ter momentos de lazer ou até mesmo descansar ${ }^{12}$.

Compreende-se que não existe certo ou errado quando o assunto é maternidade, cada mulher tem sua peculiaridade ao exercer esta função com o intuito de desfrutar de um ambiente harmônico ${ }^{11}$.

\section{Conclusão}

A partir dos resultados, constatou-se que as dificuldades são reais e há inúmeras mães/mulheres com sobrecarga física e mental.

As atividades que faziam parte da rotina da população, especificamente às mães/mulheres, devido às medidas cabíveis de proteção contra a COVID-19, foram suspensas por tempo indeterminado, forçando a necessidade de novas rotinas, as quais muitas famílias não estavam preparadas.

Verificou-se a importância de um olhar atento para mães/mulheres que vivenciam a pandemia, as quais tentam conciliar maternidade com outros tantos afazeres, acentuando uma sobrecarga.

Como estratégias para vivenciar esse momento sem comprometer o campo afetivo-emocional do binômio evidencia-se:

- Solicitar ajuda de familiares;

- Divisão de tarefas e afazeres domésticos;

- Estipular uma rotina com atividades e tarefas junto às crianças;

- Apoio social;

- Técnicas de relaxamento e respiração;

- Atividade física ou lazer;

- Manter contato on-line com amigos/familiares;

- Alimentação equilibrada;

- Atendimentos on-line;

- Partilhar relatos de experiência;

- Checar informações de fontes confiáveis sobre a COVID-19;

- Estabelecer uma pausa, mesmo que breve, para descanso;

- Divulgação para estratégias para manutenção da saúde.

As consequências oriundas do isolamento social sobre o bem-estar psicológico e o ato de maternar, em meio ao caos da pandemia, devem ser melhor exploradas, com o intuito de incentivar, bem como adotar estratégias para o desenvolvimento e a implementação de políticas públicas com ações voltadas para a maternidade, com ênfase, na prevenção e promoção da saúde física e mental às mães em momentos como este.

O cenário pandêmico atual, como se se observa nesse estudo, reforça e evidencia ainda mais a valorização da maternidade. 


\section{Referências}

1. Correia MJ. Sobre a maternidade. Análise Psicológica. 1998;3(XVI):365-371.

2. Oliveira AC, Lucas TC, Iquiapaza RA. O que a pandemia da COVID-19 tem nos ensinado sobre adoção de medidas de precaução? Text Context Enferm. 2020;29:e20200106. https://doi.org/10.1590/1980-265X-TCE-2020-0106

3. Aquino TF, Teixeira Júnior RM, José ESS, Silva JDD. Pandemia de COVID- 19: o olhar da população em relação às medidas preventivas. Glob Acad Nurs. 2020;1(3):e43. https://dx.doi.org/10.5935/2675-5602.20200043

4. Aiello-Vaisberg TMJ, Gallo-Belluzzo SR, Visintin CDN. Maternidade e sofrimento social em tempos de COVID-19: estudo de Mommy Blogs. Scielo preprints. 2020.

5. Ministério da Saúde (BR). O que é COVID-19 [Internet]. 2020 [acesso em 18 jul 2020]. Disponível em: https://coronavirus.saude.gov.br/sobre-a-doenca\#transmissao

6. Costa LM, Merchan-Hamann E. Pandemias de influenza e a estrutura sanitária brasileira: breve histórico e caracterização dos cenários. Rev Pan-Amaz Saude [Internet]. 2016 mar;7(1):11-25. http://dx.doi.org/10.5123/s2176-62232016000100002

7. Oliveira AL. A espacialidade aberta e relacional do lar: a arte de conciliar maternidade, trabalho doméstico e remoto na pandemia de COVID19. Rev. Tamoios. 2020 mai;16(1):154-166.

8. Insfran FFN, Muniz AGCR. Maternagem e COVID-19: desigualdade de gênero sendo reafirmada na pandemia. Diversitates Int. J. [Internet]. 2020 jun/dez [acesso em 24 abr 2021];12(2):26-47. Disponível em: http://www.diversitates.uff.br/index.php/1diversitatesuff1/article/view/314/248

9. Rapoport A, Piccinni CA. Maternidade e situações estressantes no primeiro ano de vida do bebê. Psico-USF. 2011 mai/ago;16(2):215-225. https://doi.org/10.1590/S1413-82712011000200010

10. Vieira PR, Garcia LP, Maciel ELN. Isolamento social e o aumento da violência doméstica: o que isso nos revela? Rev Bras Epidemiol. 2020;23:e200033. https://doi.org/10.1590/1980-549720200033

11. Visintin CDN, Gallo-Belluzzo SR, Aiello-Vaisberg TMJ. Maternidade em tempos de COVID-19: O que podemos fazer? PUC-Minas [Internet]. 2020 [acesso em 24 abr 2021]. Disponível em: http://www.puc-campinas.edu.br/wp-content/uploads/2020/05/Cartilha-Carlos-Sueli-eTa\%CC\%82nia-30abril2020-1.pdf

12. Macêdo S. Ser mulher trabalhadora e mãe no contexto da pandemia COVID-19: tecendo sentidos. Rev. Nufen: Phenom. Interd. 2020 mai/ago;12(2):187-204. http://dx.doi.org/10.26823/RevistadoNUFEN.vol12.n\%C2\%BA02rex.33

13. Moraes RF. Prevenindo conflitos sociais violentos em tempos de pandemia: garantia da renda, manutenção da saúde mental e comunicação efetiva (Nota Técnica 27). Ipea: Diretoria de Estudos e Políticas do Estado, das Instituições e da Democracia; 2020.

14. Pereira MD, et al. A pandemia de COVID-19, o isolamento social, consequências na saúde mental e estratégias de enfrentamento: uma revisão integrativa. RSD;2020;9(7):e652974548. https://doi.org/10.33448/rsd-v9i7.4548

15. Schmidt B, et al. Saúde mental e intervenções psicológicas diante da pandemia do novo coronavírus (COVID-19). Estud. psicol. 2020;37:e200063. https://doi.org/10.1590/1982-0275202037e200063

16. Fabbro MRC, Heloani JRM. Mulher, maternidade e trabalho acadêmico. Invest. educ. enferm [Internet]. 2010 jul/dez [acesso em 24 abr 2021];28(2):176-186. Disponível em: http://www.scielo.org.co/scielo.php?script=sci_arttext\&pid=S0120-53072010000200004 